\title{
Emotional and behavioural dysregulation in children of health care workers in the frontline of COVID-19 response in Sri Lanka
}

\author{
LC Rathnayake, M Chandradasa
}

\section{Abstract}

A novel coronavirus infection (COVID-19) causing respiratory failure was recognised in Wuhan China and the World Health Organization declared the infection a pandemic. Thousands of lives have been lost and the health care workers working in the frontlines are susceptible to morbidity and mortality due to the highly contagious virus. Significant psychological consequences such as sleep disturbance, anxiety, depressive symptoms, somatization, and obsessive-compulsive symptoms in health workers have been reported from China. We were unable to access any reports of mental health consequences in children of health workers. Parental psychological distress is known to affect children's mental wellbeing significantly and we report three children presenting with emotional and behavioural dysregulation whose parents are frontline COVID-19 health care workers in Sri Lanka.

Key words: Corona virus, pandemics, child, Sri Lanka, health workforce

SL J Psychiatry 2020; 11(1): 58-60

\section{Background}

A novel coronavirus infection causing significant morbidity and mortality was recognised from the Chinese city of Wuhan in December 2019. It spread across continents and was termed severe acute respiratory syndrome coronavirus 2 (COVID-19) (1). The World Health Organisation declared the infection a pandemic and thousands of lives have been lost due to the related respiratory complications (2). Health care workers working in the frontlines of the COVID-19 response are susceptible to morbidity and mortality as the virus is highly contagious (3).

Already significant mental health effects in health workers have been reported from China. An online survey conducted among more than 2000 medical and nonmedical health workers in China showed that insomnia, anxiety, depression, somatization, and obsessivecompulsive symptoms were higher in medical than nonmedical categories (4). However, we could not access any reports of psychological consequences in children of health workers.

A recent Sri Lankan study has shown that parental psychological ailments such as maternal depressive disorder could lead to hyperactivity, conduct, emotional and peer problems in children and adolescents (5). Therefore, psychological stress in parents could be considered as a risk factor for mental health issues in children. Here, we report three children of health workers, without prior history of mental health contact presenting with significant emotional and behavioural dysregulation while their parents are working in the frontline of the COVID-19 response.

\section{Case Reports}

The first was a 10-year-old boy, a son of a nursing officer working in a teaching hospital. The mother was unable to come home for a week as she worked in the ward dedicated to patients suspected of COVID-19. After returning home, she has noticed her son was experiencing sleep disturbance with frequent nocturnal awakenings, nightmares and difficulty in falling asleep at the initiation. He also had recurrent episodes of chest tightness and breathing difficulty suggestive of panic attacks. The parents were extremely concerned with this presentation that had lasted a week, where the boy repeatedly stated his fears that his mother would die of the virus. There was no prior history of mental health contact or long-term medical disorders. His physical examination and electrocardiogram were normal. He was 
treated successfully with psychoeducation, progressive muscle relaxation and advice on sleep hygiene.

The second was a 6-year-old girl, a daughter of a male medical officer working in a specialised infectious disease unit. The child was aware that her father was working at a high-risk station where new patients are admitted with the spreading disease. She presented with new-onset temper tantrums for two weeks where she was screaming and rolling on the floor, demanding that her father to stay home, when he was about to leave for work. As a result, her father started staying continuously at the hospital quarters to avoid such situations, even when there were no duties allocated to him. The child had no reported past significant psychological issues. This girl was treated with deep breathing exercises such as belly breathing technique for relaxation, and parental training on differential reinforcement. Her symptoms improved gradually with these measures.

The third was a 9-year-old boy, a son of a female specialist medical officer working in a provincial general hospital. He presented with irritability and oppositional defiance in the form of vindictive and argumentative behaviour for two weeks duration. There had been no such behaviour in the past. He was usually looked after by his father during weekdays, as his mother stayed in the hospital quarters. He demanded that his mother to take leave from work, as he feared she would contract the virus. The child had no prior history of contact with mental health services. His mother was extremely concerned as she was unable to take days off due to her altered work commitments related to the COVID-19 response. This 9-year-old boy was treated with guided imagery, including the magic shell meditation technique for relaxation and a collaborative problem-solving approach. His irritability and oppositional behaviour reduced with treatment.

Written informed consent from a parent and assent from the child were obtained. All children were assessed and treated by a consultant child and adolescent psychiatrist with training in child psychotherapy.

\section{Discussion}

The COVID-19 pandemic has caused significant morbidity and mortality worldwide. Recent research in China has shown that frontline health care workers are susceptible to significant mental health issues (4). Parents' psychological status is known to affect the children's mental wellbeing $(5,6)$. However, we could not access any reports of children of health staff affected psychologically during the pandemic. We have described three Sri Lankan children whose parents are health care workers in the frontline of the COVID-19 response presenting with significant emotional and behavioural dysregulation.
The first child presented with new-onset anxiety-related sleep disturbance and panic symptoms as his mother, a nurse, worked in a unit dedicated to suspected COVID19 patients. Reviews of the existing literature show that there is a reciprocal relationship between sleep and emotional regulation in children, and sleep disturbances are highly prevalent in children with anxiety disorders (7). The 10-year-old boy was treated with progressive muscle relaxation training, and there is past evidence that this method is effective in reducing emotional distress in 10-11-year-old children (8). The 6-year old girl presented with recent onset temper tantrums in the context of her father leaving to work in a high-risk unit managing patients with the COVID-19 infection. Short lasting temper tantrums between the ages of 2 to 6 years is a well-recognised presentation and most follow parental noncompliance to their demands (9). She was managed with differential reinforcement, which has shown to be effective in reducing temper tantrums in this age (10).

The 9-year-old boy presented with irritability and oppositional defiance as his mother was engaged in the COVID-19 health response. In a prior study among children and adolescents between the ages of 7 to 19 years, it was shown that there is a direct link between child anxiety and irritability (11). He was treated with guided imagery leading to reduction of his symptoms. Relaxation using guided imagery has shown to reduce perioperative anxiety in children between 6-12-years (12). The collaborative problem-solving approach was used to manage this 9-year-boy and this method has been used to treat oppositional behaviour in children (13). It differs from standard behavioural techniques, where we provide incentives for meeting parent expectations. This method focuses on identifying and improving the lack of cognitive skills that interfere with a child's ability to meet these expectations (13). A limitation of the described cases was that these children may have been also affected by staying home for long periods due to the curfew imposed in the country to control the spread of COVID-19.

Sri Lanka has a limited number of child mental health experts and services $(14,15)$. Innovative techniques need to be employed to maximise service provision in crises. In the aftermath of the Easter sunday bombings in 2019, social media platforms were used to provide psychoeducation to parents and teachers on the mental wellbeing in children, and non-expert health staff were trained to provide psychological support (16). Similar methods could be used to support children of frontline health workers during the COVID-19 response as well. The mental wellbeing of frontline health staff and their families is vital to allow them to continue their service during this pandemic. The mental health service providers need to collaborate with paediatric and education services to make use of limited resources and support these health care workers who are saving lives (17). 


\section{Conflicts of interest}

None declared.

LC Rathnayake, Department of Psychiatry, Faculty of Medicine, University of Kelaniya, Ragama, Sri Lanka

M Chandradasa, Department of Psychiatry, Faculty of Medicine, University of Kelaniya, Ragama, Sri Lanka, Child \& Adolescent Mental Health Services, Colombo North Teaching Hospital, Ragama, Sri Lanka, and Faculty of Medicine Nursing \& Health Sciences, Monash University, Clayton, Australia

Corresponding author: $\mathrm{M}$ Chandradasa

Email: miyuruc@kln.ac.lk

http://orcid.org/0000-0002-1873-8228

\section{References}

1. Li Q, Guan X, Wu P, et al. Early Transmission Dynamics in Wuhan, China, of Novel Coronavirus-Infected Pneumonia. N Engl J Med. 2020; 382(13): 1199-207. doi:10.1056/NEJMoa2001316

2. World Health Organisation (WHO). 2020. Rolling Updates on Coronavirus Disease (COVID-19). URL https:// www.who.int/emergencies/diseases/novel-coronavirus2019/events-as-they-happen (Accessed 4.19.20)

3. Kooraki S, Hosseiny M, Myers L, Gholamrezanezhad A. Coronavirus (COVID-19) Outbreak: What the Department of Radiology Should Know. J Am Coll Radiol. 2020; 17(4): 447-451. doi:10.1016/j.jacr.2020.02.008

4. Zhang WR, Wang K, Yin L, et al. Mental Health and Psychosocial Problems of Medical Health Workers during the COVID-19 Epidemic in China [published online ahead of print, 2020 Apr 9]. Psychother Psychosom. 2020; 1-9. doi:10.1159/000507639

5. Rohanachandra YM, Prathapan S, Wijetunge S. Psychological Problems and Socioemotional Wellbeing among Children of Mothers with Depression and Their Association with Sociodemographic Factors in a Sri Lankan setting. Psychiatry J. 2018; 2018: 3809384. Published 2018 Apr 16. doi:10.1155/2018/3809384

6. Eruyar S, Maltby J, Vostanis P. Mental health problems of Syrian refugee children: the role of parental factors. Eur Child Adolesc Psychiatry. 2018; 27(4) :401-9. doi:10.1007/ s00787-017-1101-0

7. Brown WJ, Wilkerson AK, Boyd SJ, Dewey D, Mesa F, Bunnell BE. A review of sleep disturbance in children and adolescents with anxiety. J Sleep Res. 2018; 27(3): e12635. doi:10.1111/jsr.12635
8. Hashim HA, Zainol NA. Changes in emotional distress, short term memory, and sustained attention following 6 and 12 sessions of progressive muscle relaxation training in 10-11 years old primary school children. Psychol Health Med. 2015; 20(5): 623-8. doi:10.1080/13548506. 2014.1002851

9. Eisbach SS, Cluxton-Keller F, Harrison J, Krall JR, Hayat M, Gross D. Characteristics of temper tantrums in preschoolers with disruptive behaviour in a clinical setting. J Psychosoc Nurs Ment Health Serv. 2014; 52(5): 32-40. doi:10.3928/02793695-20140110-02

10. Wilder DA, Chen L, Atwell J, Pritchard J, Weinstein P. Brief functional analysis and treatment of tantrums associated with transitions in preschool children. J Appl Behav Anal. 2006; 39(1): 103-7. doi:10.1901/jaba/ 2006.66-04

11. Cornacchio D, Crum KI, Coxe S, Pincus DB, Comer JS. Irritability and Severity of Anxious Symptomatology Among Youth with Anxiety Disorders. J Am Acad Child Adolesc Psychiatry. 2016; 55(1): 54-61. doi:10.1016/ j.jaac.2015.10.007

12. Vagnoli L, Bettini A, Amore E, De Masi S, Messeri A. Relaxation-guided imagery reduces perioperative anxiety and pain in children: a randomized study. Eur J Pediatr. 2019; 178(6): 913-921. doi:10.1007/s00431-019-03376-х

13) Greene RW, Ablon JS, Goring JC. A transactional model of oppositional behaviour: underpinnings of the Collaborative Problem-Solving approach. J Psychosom Res. 2003; 55(1): 67-75. doi:10.1016/s0022-3999(02) 00585-8

14. Chandradasa M, Kuruppuarachchi KALA. Child and youth mental health in post-war Sri Lanka. BJPsych Int. 2017; 14(2): 36-7. Published 2017 May 1. doi:10.1192/ s2056474000001756

15. Chandradasa M, Champika L. Subspecialisation in Postgraduate Psychiatry and Implications for a ResourceLimited Specialised Child and Adolescent Mental Health Service. Acad Psychiatry. 2019; 43(1): 135-9. doi:10.1007/ s40596-018-0920-8

16. Chandradasa M, Rathnayake LC, Rowel M, Fernando L. Early phase child and adolescent psychiatry response after mass trauma: Lessons learned from the Easter Sunday attack in Sri Lanka [published online ahead of print, 2020 Mar 20]. Int J Soc Psychiatry. 2020; 20764020913314. doi:10.1177/0020764020913314

17. Chandradasa M, Basu S. Collaborative networking between regional child mental health, paediatric and educational services in Gippsland, Australia: An online survey. Aust J Rural Health. 2019; 27(6): 571-3. doi:10.1111/ajr.12552 